\title{
Near-Field Focusing With Subwavelength Thickness Metalenses via Electromagnetic Susceptibility Models
}

\author{
Zhenguan Fu \\ College of Science, University of Shanghai for Science and Technology, Shanghai, China \\ Email: 441097901@qq.com
}

How to cite this paper: Fu, Z.G. (2021) Near-Field Focusing With Subwavelength Thickness Metalenses via Electromagnetic Susceptibility Models. Optics and Photonics Journal, 11, 197-209.

https://doi.org/10.4236/opj.2021.117014

Received: June 4, 2021

Accepted: July 13, 2021

Published: July 16, 2021

Copyright $\odot 2021$ by author(s) and Scientific Research Publishing Inc. This work is licensed under the Creative Commons Attribution International License (CC BY 4.0).

http://creativecommons.org/licenses/by/4.0/

\section{(c) (i) Open Access}

\begin{abstract}
The design mentality of an optimal metalens model, based on the electromagnetic susceptibility, a synthesis of subwavelength-thick metasurfaces (MSs) is presented in this paper. First, based on the finite difference method of generalized sheet transition conditions, the surface susceptibility function of the MS with spatial discontinuities can be determined. Then, the paper analyzed the remaining corresponding physical field conditions for the scale of metalens. In order to adapt to the physical limitations encountered in the near-field focusing of the metalens, a standard parabolic phase design is proposed in this paper, and its upsides and downsides of the two-phase processing in different aspects are compared. Using COMSOL software with numerical simulation, it can be seen that the standard design can easily obtain high resolution in the near field, while the focusing effect is more stable when the focal length is small by the parabolic phase design.
\end{abstract}

\section{Keywords}

GSTCs, Metalens, Near-Field Focusing, Susceptibility

\section{Introduction}

The lens is the most basic element of the optical system, and its history can even be traced back to the ancient crystal ball used for divination and "seeing". Today, as a device that converges or diffuses electromagnetic radiation, it is widely used in various fields such as optical instruments, cameras, security, and vehicles. Traditional lenses realize light convergence and divergence by adjusting the optical path of light with different wavelengths. Common optical instruments always use multiple curved lenses with different thicknesses and materials to focus 
images. Inevitably, this increases the scale of the instruments' volume. However, in the face of increasing requirements of imaging with higher standards, it is difficult for traditional lenses to be designed to be thinner and more compact [1], which is the reason that manufacturers have made large magnification microscopes and telephoto lenses so bloated.

A metalens is a planar optical device that uses a combination of artificial sub-wavelength units to perform wave-front manipulation. Different from the traditional lens, the metalens is processed by the increasingly mature nanofabrication technology, the field intensity and phase of the light wave are modulated through the surface nano structure. Therefore, different functions can be realized by setting the properties of each nano-element and its spatial position, without the need for size or curved surface to accumulate appropriate propagation phase as well as complex lens group in a view to achieving electromagnetic wave manipulation [2]. The Huygens metasurface unit can be designed to have an ultra-high transmission amplitude (maximum close to 100\%) [3]. Han Lin et al. even used a femtosecond laser to process an ultra-thin flat lens in a two-dimensional single-atom layer with a thickness of only 7 angstroms. It has high-quality imaging capabilities, including sub-wavelength resolution and $31 \%$ focusing efficiency [4]. The metalens has remarkable characteristics such as aberration correction, active tunability and translucency [5], especially for near-field wavefront control, it has natural advantages (higher numerical aperture, ultra-high resolution). Near-field focusing imaging using surface waves has already been applied to a variety of near-field scanning scenes due to its extremely small half-width of the focal peak [6] [7] [8] [9]. But these lenses are often difficult to process with complex structure, or adapt to flexible design requirements. However, the Huygens metalens can precisely control and shape the amplitude, phase and polarization state of the electromagnetic wave front [10], it provides unprecedented design flexibility and performance for the near-field focusing of the radiation wave.

This article explains in detail the metalens synthesis method based on Huygens principle, and distinguishes the pros and cons (flexibility to Angle and frequency of incident light) of the two focus phase types in the near-field. Finally, it will introduce the improvement of the susceptibility function of metalens synthesis under some design requirements, and expand some ideas for metalens design.

\section{Design Principle of Metalens}

When discussing metasurface, it generally refers to a set of scatters composed of composite media (even thin-film conforming to classical theory). Just like the three-dimensional (volume) metamaterial [6], the behavior of the MSs is determined by the electrical and magnetic polarizabilities of the scattering elements. In profile, the metalens is composed of a series of specific scattering elements that closely arranged. The MS of one or several elements can be viewed as a clas- 
sical equivalent medium film in a range of deep subwavelength dimensions of thickness $d$ (Figure 1). Thus, the effective surface parameters are designed independent of frequency (in fact, when modeling an MS, the surface properties may be adapted to a certain frequency range), but rather take into account the effect of the specific thickness of the MS.

The effective-medium theory is the traditional and most convenient method for modeling metamaterials, yet the simple introduction of bulk parameters to the metamaterial is not accurate enough to the electromagnetic discontinuity of the metasurface boundary [7] [8] [9]. It has been shown that a type of boundary conditions known as generalized sheet transition conditions (GSTCs) are the most appropriate way for scattering by MSs [8] [9] [10] [11]. It refers to the use of the equivalent surface parameters of the MS (susceptibility etc.) under equivalent boundary conditions, and these parameters are the all required to model the macroscopic interaction between any given MS and the electromagnetic field. Based on the Huygens principle, this paper equates an MS to a set of Huygens sources [12], exactly consistent with GSTC. It needs rigorous GSTCs to properly handle the discontinuity [13] [14] on the distribution. The metalens is based on the description metasurface by the susceptibility tensor in space, associated to the incident, reflection and transmission fields on both sides of the structure via GSTC (Figure 2). The corresponding states between the MS polarization densities and the nearby effective electromagnetic field

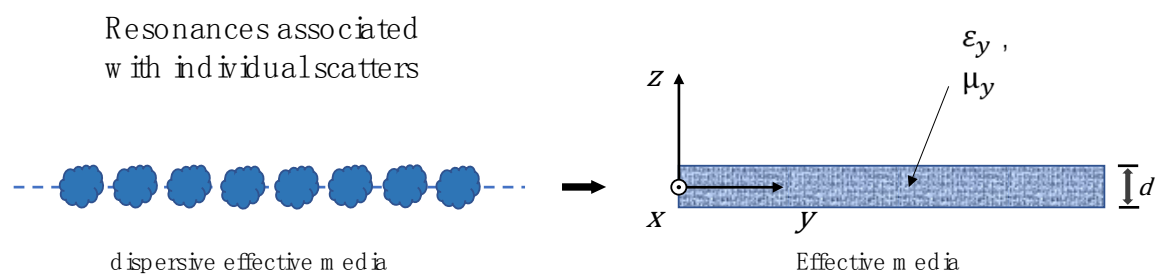

Figure 1. Metasurface is regarded as an effective medium with thickness $d$.

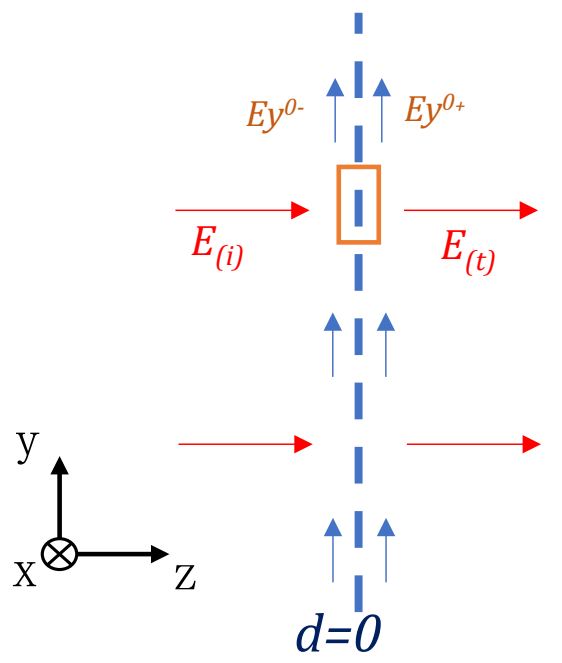

Figure 2. Schematic diagram of the electric field in the case of MS GSTCs with thickness of 0 . 


$$
\begin{gathered}
\hat{z} \times \Delta \boldsymbol{H}=j \omega \boldsymbol{P}_{\|}-\hat{z} \times \nabla_{\|} M_{z}, \\
\Delta \boldsymbol{E} \times \hat{z}=j \omega \mu \boldsymbol{M}_{\|}-\nabla_{\|}\left(\frac{P_{z}}{\varepsilon}\right) \times \hat{z} .
\end{gathered}
$$

For the convenience of closed form solutions, paper makes $P_{z}=M_{z}=0, \mathrm{P}$ and $M$ are electric and magnetic polarization densities, respectively, then can be expressed as

$$
\begin{aligned}
& \boldsymbol{P}=\varepsilon \overline{\bar{\chi}}_{e e} \boldsymbol{E}_{a v}+\overline{\bar{\chi}}_{e m} \sqrt{\mu \varepsilon} \boldsymbol{H}_{a v}, \\
& \boldsymbol{M}=\overline{\bar{\chi}}_{m m} \boldsymbol{H}_{a v}+\overline{\bar{\chi}}_{m e} \sqrt{\frac{\varepsilon}{\mu}} \boldsymbol{E}_{a v} .
\end{aligned}
$$

Here the solution is expressed in terms of the transverse susceptibility functions, $\overline{\bar{\chi}}_{e e}, \overline{\bar{\chi}}_{e m}, \overline{\bar{\chi}}_{m m}, \overline{\bar{\chi}}_{m e}$, which represent the electric/magnetic $(e / m)$ transverse polarization responses (first subscript) to transverse electric/magnetic $(e / m)$ field excitations (second subscript). $\Psi_{a v}=\frac{\left[\Psi_{t}+\left(\Psi_{r}+\Psi_{i}\right)\right]}{2}, \Psi=\boldsymbol{H}, \boldsymbol{E}$. Substituting the formula (2) into the formula (1), it can obtain the constraint condition of the susceptibility,

$$
\begin{aligned}
& \left(\begin{array}{c}
-\Delta H_{y} \\
\Delta H_{x}
\end{array}\right)=j \omega \varepsilon\left(\begin{array}{cc}
\chi_{e e}^{x x} & \chi_{e e}^{x y} \\
\chi_{e e}^{y x} & \chi_{e e}^{y y}
\end{array}\right)\left(\begin{array}{l}
E_{x, a v} \\
E_{y, a v}
\end{array}\right)+j \omega \sqrt{\varepsilon \mu}\left(\begin{array}{cc}
\chi_{e m}^{x x} & \chi_{e m}^{x y} \\
\chi_{e m}^{y x} & \chi_{e m}^{y y}
\end{array}\right)\left(\begin{array}{l}
H_{x, a v} \\
H_{y, a v}
\end{array}\right), \\
& \left(\begin{array}{c}
\Delta E_{y} \\
-\Delta E_{x}
\end{array}\right)=j \omega \mu\left(\begin{array}{ll}
\chi_{m m}^{x x} & \chi_{m m}^{x y} \\
\chi_{m m}^{y x} & \chi_{m m}^{y y}
\end{array}\right)\left(\begin{array}{l}
H_{x, a v} \\
H_{y, a v}
\end{array}\right)+j \omega \sqrt{\varepsilon \mu}\left(\begin{array}{cc}
\chi_{m e}^{x x} & \chi_{m e}^{x y} \\
\chi_{m e}^{y x} & \chi_{m e}^{y y}
\end{array}\right)\left(\begin{array}{l}
E_{x, a v} \\
E_{y, a v}
\end{array}\right) .
\end{aligned}
$$

To simplify the model, it considers here the design of a single-refraction lens. The MS with no thickness is placed at $z=0$, and the light enters from one side $\left(\overline{\bar{\chi}}_{e m} \equiv \overline{\bar{\chi}}_{m e}=0\right.$ and diagonal $\left.\chi_{e e}^{x y} \equiv \chi_{e e}^{y x} \equiv \chi_{m m}^{x y} \equiv \chi_{m m}^{y x}=0\right)$ [15]. Four expressions of polarization coefficients can be obtained from Equation (3):

$$
\begin{aligned}
\chi_{e e}^{\chi x} & =\frac{-\Delta H_{y}}{j \omega \varepsilon E_{x, a v}}, \\
\chi_{e e}^{y y} & =\frac{\Delta H_{x}}{j \omega \varepsilon E_{y, a v}}, \\
\chi_{m m}^{x x} & =\frac{\Delta E_{y}}{j \omega \mu H_{x, a v}}, \\
\chi_{m m}^{y y} & =\frac{-\Delta E_{x}}{j \omega \mu H_{y, a v}} .
\end{aligned}
$$

In order to get a lossless $(k=\omega \sqrt{\mu \varepsilon})$ and non-reflective metalens, $A^{t}=A^{i}$, $\left\{\begin{array}{l}\boldsymbol{E}^{r}=0 \\ \boldsymbol{H}^{r}=0\end{array}\right.$. For Huygens type MS, the transmitted electromagnetic wave is $\left\{\begin{array}{l}\boldsymbol{E}^{t}=\boldsymbol{E}^{i} \mathrm{e}^{j \varphi_{t}} \\ \boldsymbol{H}^{t}=\boldsymbol{H}^{i} \mathrm{e}^{j \varphi_{t}}\end{array}\right.$, where the $\varphi_{t}$ is retardation imparted by MS, the transmission coefficient is $T_{x}=\boldsymbol{E}_{x}^{t} / \boldsymbol{E}_{x}^{i}=\mathrm{e}^{j \varphi_{t}}$, in TM mode, 


$$
\begin{aligned}
& \Delta E_{x}=A^{i}\left(\mathrm{e}^{j \varphi_{t}}-1\right), \\
& \Delta H_{y}=\frac{A^{i}}{\eta}\left(\mathrm{e}^{j \varphi_{t}}-1\right), \\
& E_{x, a v}=\frac{A^{i}}{2}\left(\mathrm{e}^{j \varphi_{t}}+1\right), \\
& H_{y, a v}=\frac{A^{i}}{2 \eta}\left(\mathrm{e}^{j \varphi_{t}}+1\right) .
\end{aligned}
$$

the same in TE mode. Substituting Equation (5) into Equation (4), it can finally obtain the electric/magnetic polarization coefficient

$\chi=\chi_{e e}^{x x}=\chi_{m m}^{y y}=\chi_{m m}^{x x}=\chi_{e e}^{y y}=\frac{2 j}{k}\left(\frac{T_{x}-1}{T_{x}+1}\right)$.

Nanfang $\mathrm{Yu}$ and Capasso et al. have produced a variety of superior superlenses with excellent performance [16]-[21] and Carl Pfeiffer et al. have experimentally demonstrated the non-reflective Huygens metasurface [22] that provides a space-varying phase response by radio frequencies-microwaves. They all considered building a spherical phase profile to obtain the focus.

In general, for a metalens with a given focal length $f$, the phase shift of transmitted light at an arbitrary point $m$ on the profile needs to be adjusted $\Delta \varphi_{m}$ (Figure 3), and the equation is satisfied

$$
\Delta \varphi_{m}=k \cdot \overline{m Q}=\frac{2 \pi}{\lambda}\left(\sqrt{\left(y_{m}^{2}+f^{2}\right)}-f\right)
$$

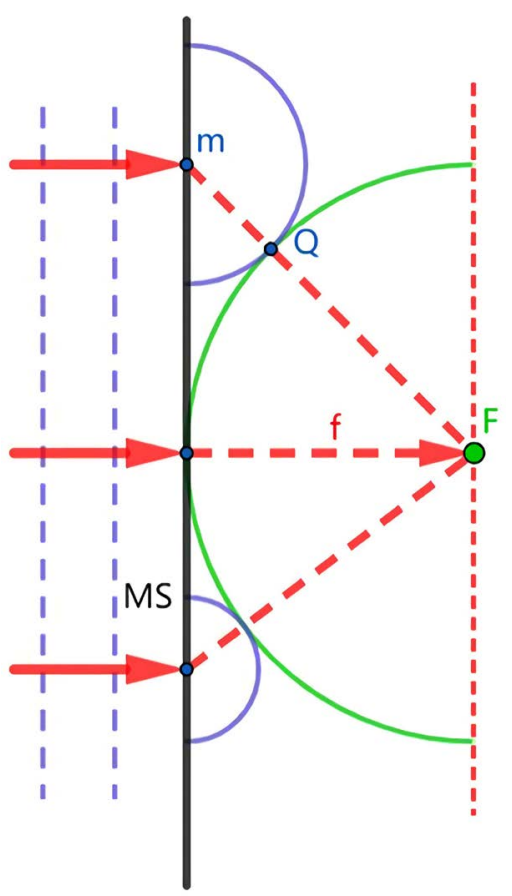

Figure 3. The image shows a two-dimensional profile of a general Huygens metasurface focusing. The light at the point $\mathrm{m}$ on the MS is given a specific phase shift, which forms an equal-phase plane centered on $\mathrm{F}$ together with the adjacent scatters. 
Here, the $\lambda$ is the wavelength of electromagnetic waves in free space, $y_{m}$ is the distance of the scattering element from the center of the MS. The transmitted light passing through point $\mathrm{m}$ on the MS will form a circular equal phase surface with the adjacent scattered light at point $\mathrm{Q}$ following the Huygens principle, and form the focus at point F. Hence, the control function $\chi$ about the e/m susceptibility on the MS can be obtained.

For the convenient, it is assumed that the plane wave $(100 \mathrm{GHz})$ is irradiated on the surface through vacuum from the apture at $2 \lambda$ on the left, and then focused at point $F$ in vacuum on the right after modulation. In order to ensure the focusing effect, the aperture size is limited by the scale of the focal length. In the study of near field focusing, the incident width of $8 \lambda$ is set for convenience, moreover, the MS thickness $d$ is selected as $\lambda / 10$ to satisfy the subwavelength and facilitate manufacturing (as long as $\mathrm{d}$ maintains the subwavelength, the permittivity and permeability can be effectively approximated to $\varepsilon_{r}=1+\chi_{E S} / d$, $\mu_{r}=1+\chi_{M S} / d$, respectively) [13] [23] [24]. Please see Figure 4 for details.

Although such design of Huygens metalens has the natural advantage of super-resolution in near-field focusing [25], the contribution of scatters at both ends of the MS to the focal spot is too small when the Angle of elevation is large. Besides, the characteristic of high numerical aperture and a large part of the incident light cannot be well utilized.

To this end, it optimizes the metalens based on Huygens' principle and use the isophased surface for the second time. It is assumed that the transmitted light first forms a parabolic wave front, and then forms a circular wave front, the plane wave incident to MS also coherently focuses at the point F. For convenience's sake, a special situation is selected (see Figure 5), and the phase shift of transmitted light at point $\mathrm{m}$ on the profile needs to be adjusted as follows

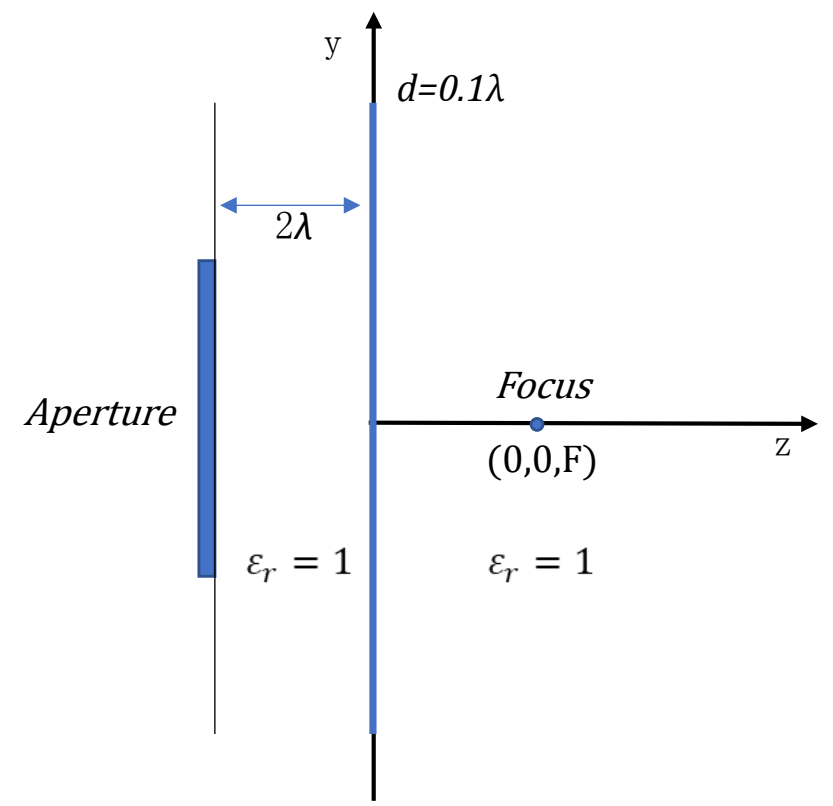

Figure 4. Diagram of conditions for study of metalens. 


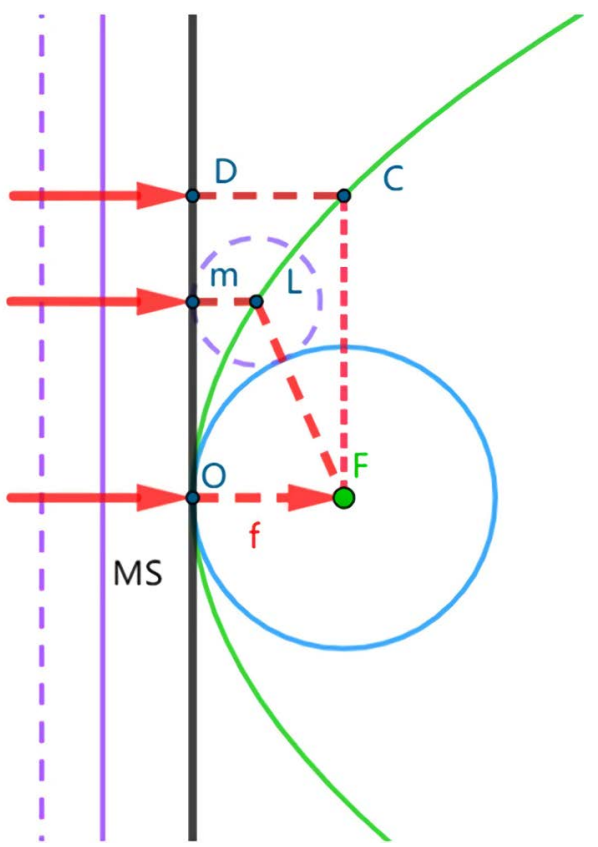

Figure 5. Schematic diagram of parabolic isophase focusing. Point D is the boundary that can contribute constraints to the parabolic focus, at this time the effective aperture $L=2 \overline{O D}=4 f$.

$$
\Delta \varphi_{m}^{\prime}=k \cdot 2 \overline{m L}=\frac{2 \pi}{\lambda} \cdot \frac{y_{m}^{2}}{2 f}
$$

Paraboloidal phase processing (PPP) requires a larger phase difference than the general focusing phase model, which results in smaller amplitude on the wave front and smaller focal spot energy. Of course, since the phase shift of adjacent elements is larger in this design, it also avoids the problem of insufficient phase shift of adjacent elements, which is easy to occur in the fabrication of MS elements [26].

\section{Analysis and Comparison of Metalens}

Based on the design method of metalens introduced above, this article uses COMSOL multiphysics simulation software to analyze its electromagnetic characteristics in detail. Just write the equivalent dielectric property $\varepsilon_{r}, \mu_{r}$ into an equal-thickness medium layer of sufficient length. Three sets of Huygens metalenses with different focal lengths $\left(f_{1}=3 \lambda, f_{2}=\lambda, f_{3}=0.5 \lambda\right)$ are given below. Since the uniform incident light width of $8 \lambda$ is set, the equivalent numerical aperture is $N_{A 1}=0.8, N_{A 2}=0.97, N_{A 3}=0.99$, respectively, as shown in Figure 6.

In order to improve the design, it uses the new parabolic phase design introduced above to compare with the old design of the metalens at a specific focal length in the near field. It can be seen from Figure 6 that when the focal length is $0.5 \lambda$, there will be obvious side-lobes near the focal spot of Huygens spherical phase focusing, while the parabolic design does not (see Figure 7). Comparing 

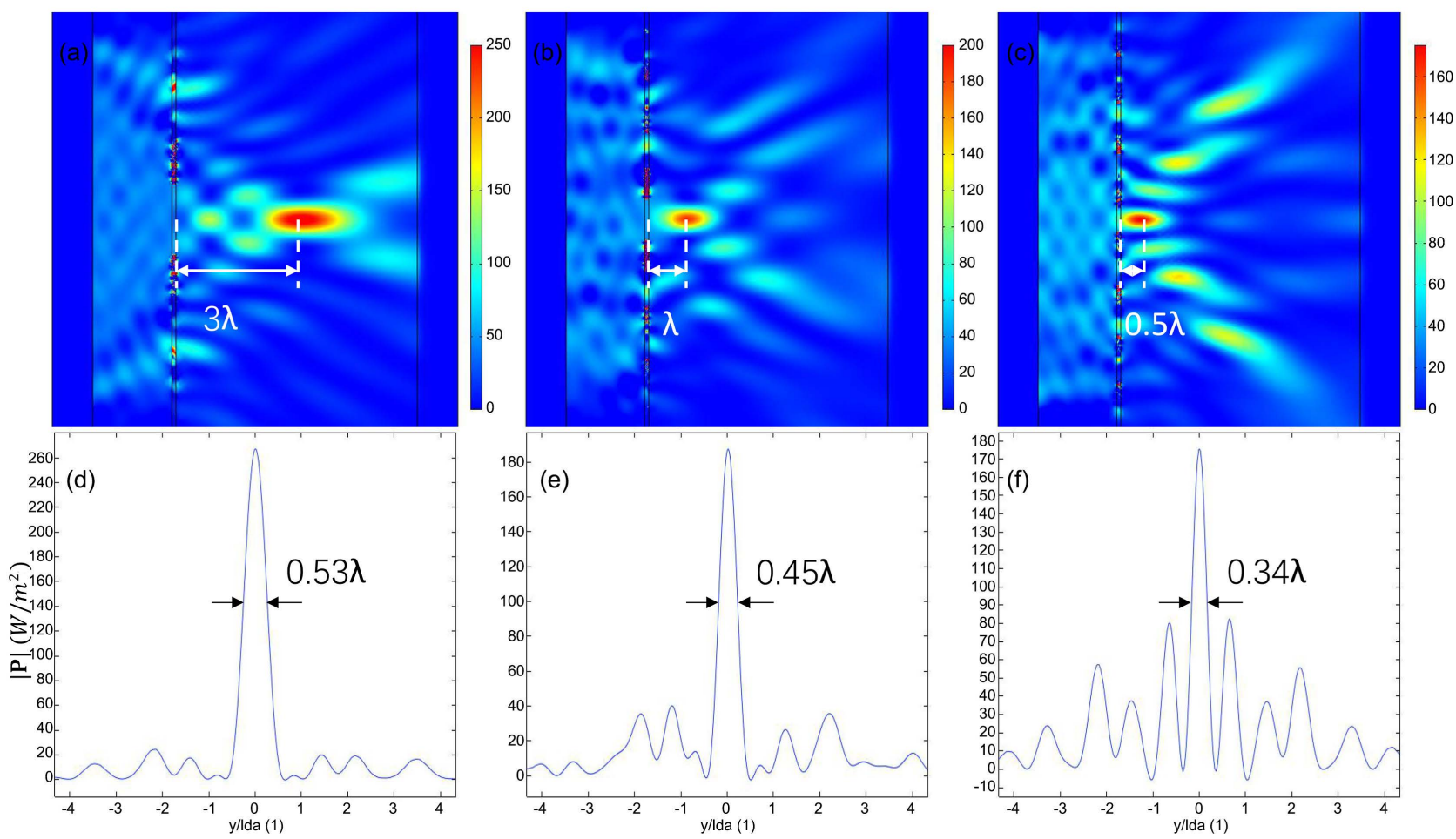

Figure 6. The energy distribution diagram of metalenses with different focal lengths $\left(f_{1}=3 \lambda, f_{2}=\lambda, f_{3}=0.5 \lambda\right)$, as show in (a), (b), (c), respectively. It can be seen from the different color legends that the focal spot intensity decreases as the focal length decreases. (d), (e), (f) are the cross-sectional views at the focal plane of the upper energy map, from which it can be seen that as the focal length decreases, the full width at half maximum decreases significantly.
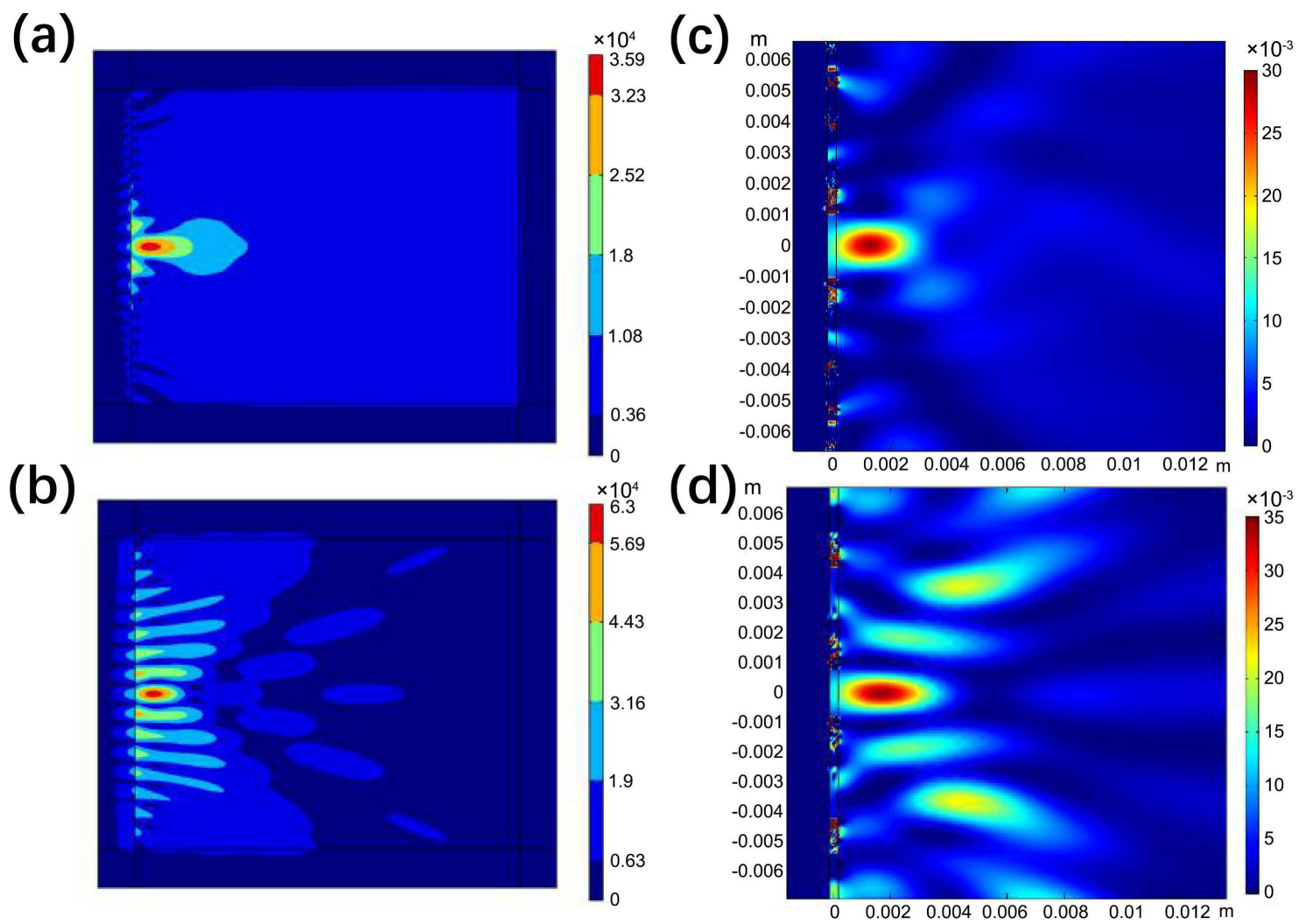
(e)
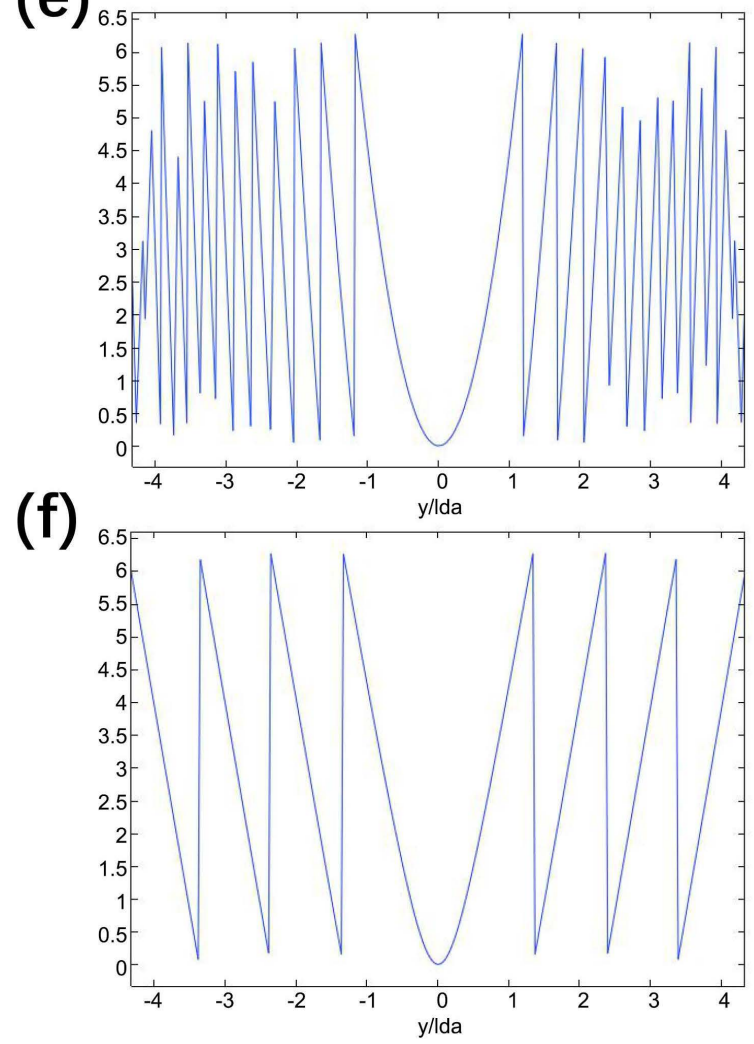

(g)

(h)
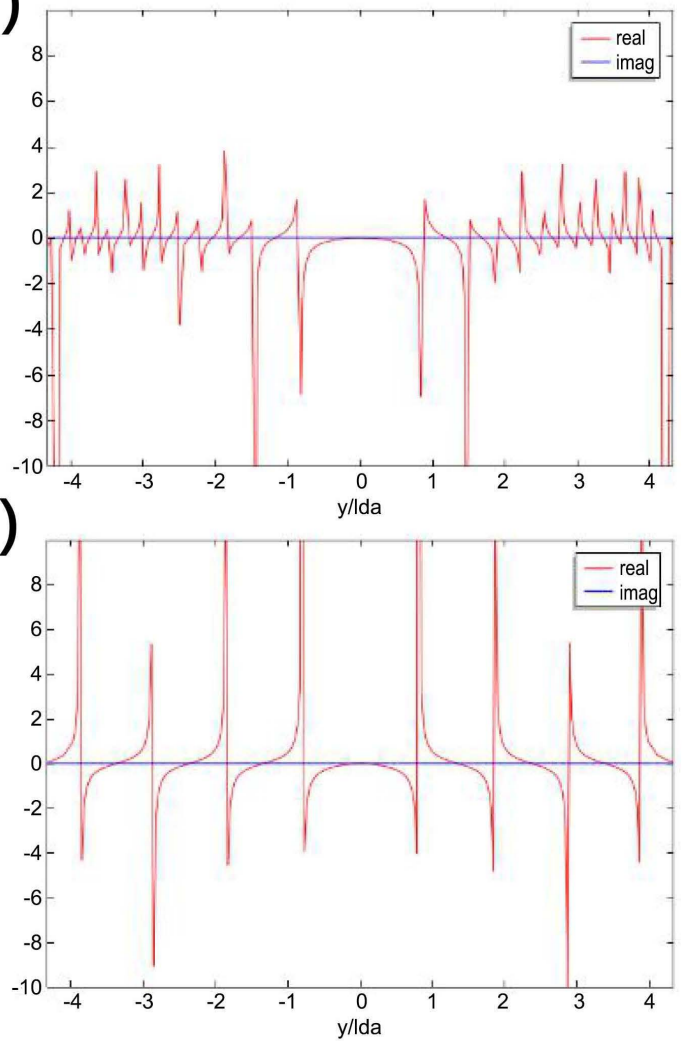

Figure 7. Figures (a) and (b) are numerical simulations of a plane wave with a parabolic phase and a circular phase, respectively. (c), (d) are the simulation solutions of the parabolic phase and the circular Huygens phase of the metalens, (e), (f) are the phase distribution diagrams of the corresponding metalens. (g), (h) are corresponding to the polarization distribution of the metalens, the $y$-axis scale is $\chi / \lambda$.

the two, the parabolic peak intensity is about half of the former (nearly double phase shift), but the contrast color legend shows that there are very few areas above the half peak and no interference peaks at all. In addition, the new phase design super lens with a focal length of $0.5 \lambda$ has a half-peak width of $0.42 \lambda$. Although the resolution has been reduced, it is still a good performance. Next, paper analyzes the adaptability of the metalens to the deflection angle of the incident light (see Figure 8). From the numerical solution, the parabolic phase has good stability for the focusing of incident light from different angles, the position of the focal plane remains basically unchanged, and the shape of the focal spot does not change much, while the circular phase focusing is quite sensitive to the incident angle. For the metalens, the parabolic design does not show the obvious advantage of the numerical solution, but it can still be seen from the comparison of the field distribution diagram that it has a better suppression effect on the messy light spots. Subsequently, it analyzed the adaptability of the two designs of the metalens to the incident frequency when the focal length is $\lambda$ and $0.5 \lambda$ (see Figure 9). This paper analyzes the focusing effect of the two designs with the axial focal shift $\pm 20 \%$ as the standard. When the focal length is $\lambda$, the performance of the two is close, and when the focal length is $0.5 \lambda$, the parabolic 
(a)

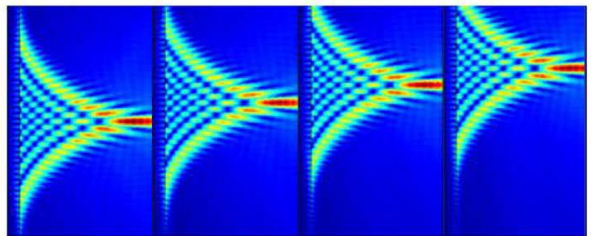

(b)

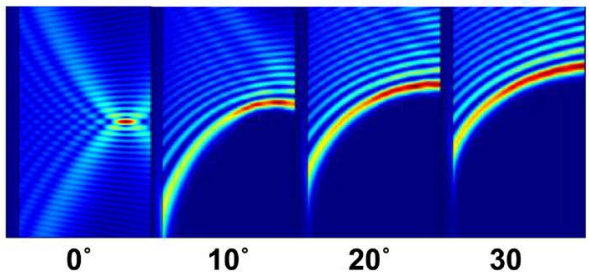

(c)

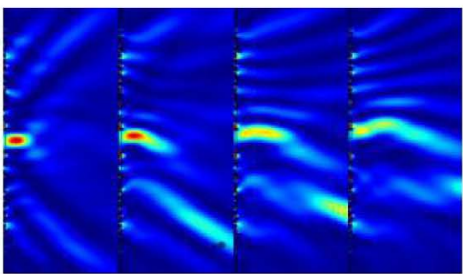

(e)

(d)
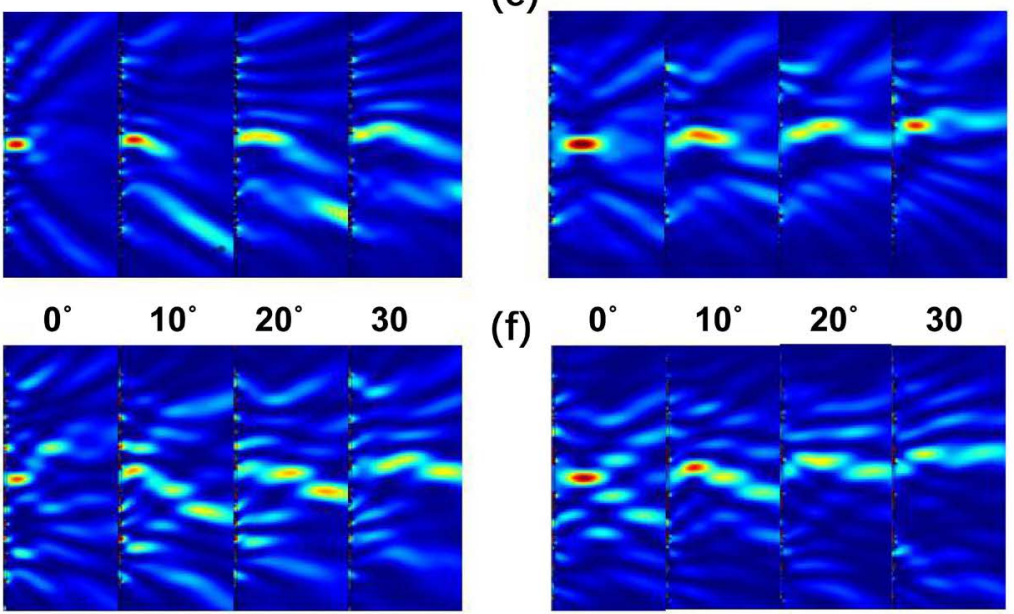

(f)

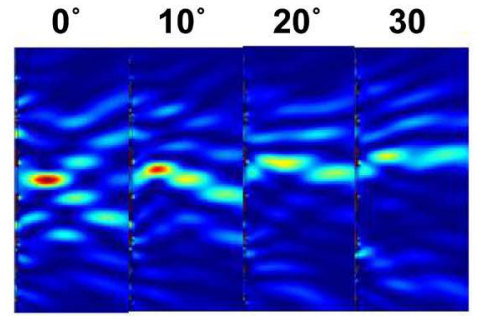

Figure 8. Figures (a) and (b) represent the ideal numerical simulation results of the parabolic phase design and the circular phase design with incidence angles from $0^{\circ}$ to $30^{\circ}$, respectively. (c), (d) are the simulation solutions of the metalens corresponding to the angle of the new design and the old phase when the focal length is $0.5 \lambda$, (e), (f) are the solutions of the metalens corresponding to the focal length of $\lambda$.

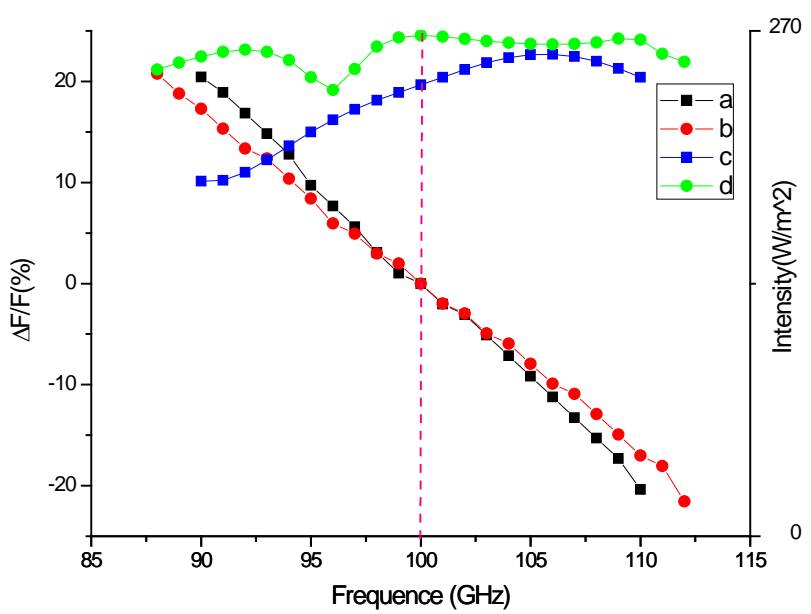

(a)

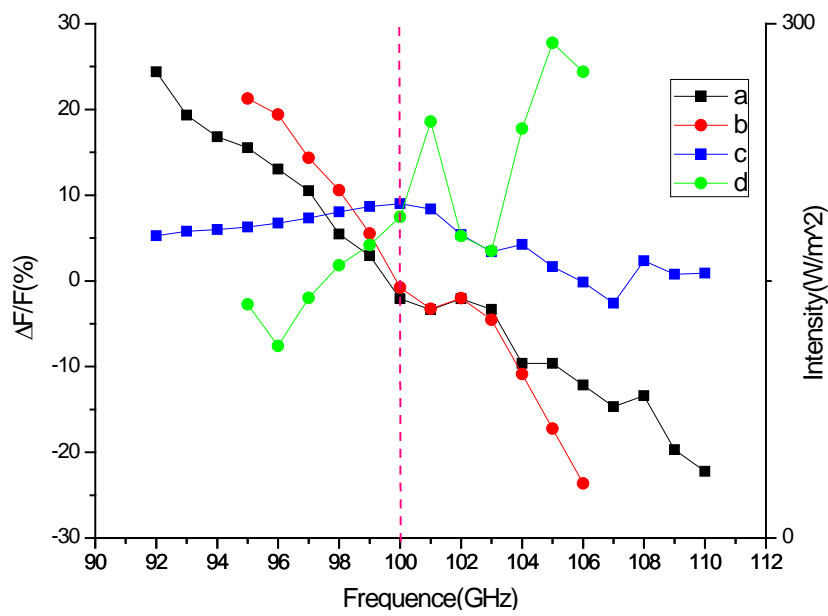

(b)

Figure 9. Figures (a) and (b) show the focal lengths are $\lambda$ and $\lambda / 2$ respectively. Curves a and $b$ represent the focal shifts of metalens of parabolic and circular designs, respectively; $c$ and $d$ represent the focal intensities of the two kinds of metasurfaces at corresponding frequencies. 
focus can adapt to a wider frequency range of focus shift, and the focal spot energy does not change much compared with the reference frequency, which is obviously better than the old design.

\section{Summary}

In this paper, the e/m susceptibility control method applies GSTC theory to design and analyze the near-field transmission and focusing characteristics of lossless subwavelength Huygens hypersurface from a two-dimensional perspective, and provides a method for solving the near-field physical limitation of the Huygens metalens. Huygens metalens can achieve a good focusing effect, and the new phase design brings more theoretical advantages (such as diameter size, incident Angle, etc.) to the near field focusing. In addition, people can also try different expressions of $e / m$ susceptibility to adapt to different design requirements, metalens has a huge application potential.

\section{Conflicts of Interest}

The author declares no conflicts of interest regarding the publication of this paper.

\section{References}

[1] Khorasaninejad, M., Chen, W., Devlin, R., Oh, J., Zhu, A. and Capasso, F. (2016). Metalenses at Visible Wavelengths: Diffraction-Limited Focusing and Subwavelength Resolution Imaging. Science, 352, 1190-1194. https://doi.org/10.1126/science.aaf6644

[2] Sun, S., He, Q., Hao, J., Xiao, S. and Zhou, L. (2019). Electromagnetic Metasurfaces: Physics and Applications. Advances in Optics and Photonics, 11, 380-479. https://doi.org/10.1364/AOP.11.000380

[3] Hao, W., Deng, M., Chen, S. and Chen, L. (2019). High-Efficiency Generation of Airy Beams with Huygens' Metasurface. Physical Review Applied, 11, Article ID: 054012. https://doi.org/10.1103/PhysRevApplied.11.054012

[4] Li, H., Xu, Z.-Q., Cao, G., Zhang, Y., Zhou, J., Wang, Z., et al. (2020) Diffraction-Limited Imaging with Monolayer 2D Material-Based Ultrathin Flat Lenses. Light. Science \& Applications, 9, Article No. 137.

https://doi.org/10.1038/s41377-020-00374-9

[5] Moon, S.W., Kim, Y., Yoon, G. and Rho, J. (2020) Recent Progress on Ultrathin Metalenses for Flat Optics. iScience, 23, Article ID: 101877. https://doi.org/10.1016/j.isci.2020.101877

[6] Maradudin, A.A. (Ed.) (2011) Structured Surfaces as Optical Metamaterials. Cambridge University Press, Cambridge.

[7] Schelkunoff, S. (1972) On Teaching the Undergraduate Electromagnetic Theory. IEEE Transactions on Education, 15, 15-25. https://doi.org/10.1109/TE.1972.4320709

[8] Holloway, C.L., Kuester, E.F., Gordon, J.A., O’Hara, J., Booth, J. and Smith, D.R. (2012) An Overview of the Theory and Applications of Metasurfaces: The Two-Dimensional Equivalents of Metamaterials. IEEE Antennas and Propagation Magazine, 54, 10-35. https://doi.org/10.1109/MAP.2012.6230714 
[9] Holloway, C.L., Kuester, E.F. and Dienstfrey, A. (2011) Characterizing Metasurfaces/Metafilms: The Connection between Surface Susceptibilities and Effective Material Properties. IEEE Antennas and Wireless Propagation Letters, 10, 1507-1511. https://doi.org/10.1109/LAWP.2011.2182591

[10] Holloway, C.L. and Kuester, E.F. (2016) A Homogenization Technique for Obtaining Generalized Sheet Transition Conditions (GSTCs) for a Metafilm Embedded in a Magneto-Dielectric Interface. IEEE Transactions on Antennas and Propagation, 64, 4671-4686. https://doi.org/10.1109/TAP.2016.2600764

[11] Holloway, C.L. and Kuester, E.F. (2017) Generalized Sheet Transition Conditions for a Metascreen-A Fishnet Maresurface. IEEE Transactions on Antennas and Propagation, 66, 2414-2427. https://doi.org/10.1109/TAP.2018.2809620

[12] Pfeiffer, C., Emani, N.K., Shaltout, A.M., Boltasseva, A., Shalaev, V.M. and Grbic, A. (2014) Efficient Light Bending with Isotropic metamaterial Huygens Surfaces. Nano Letters, 14, 2491-2497. https://doi.org/10.1021/nl5001746

[13] Idemen, M.M. (2011) Discontinuities in the Electromagnetic Field. John Wiley \& Sons, Hoboken. https://doi.org/10.1002/9781118057926

[14] Achouri, K., Salem, M. and Caloz, C. (2014). General Metasurface Synthesis Based on Susceptibility Tensors. IEEE Transactions on Antennas and Propagation, 63, 2977-2991. https://doi.org/10.1109/TAP.2015.2423700

[15] Achouri, K. and Caloz, C. (2018) Design, Concepts, and Applications of Electromagnetic Metasurfaces. Nanophotonics, 7, 1095-1116. https://doi.org/10.1515/nanoph-2017-0119

[16] Aieta, F., Genevet, P., Kats, M.A., Yu, N., Blanchard, R., Gaburro, Z., et al. (2012) Aberration-Free ultrathin Flat Lenses and Axicons at Telecom Wavelengths Based on Plasmonic Metasurfaces. Nano Letters, 12, 4932-4936.

https://doi.org/10.1021/nl302516v

[17] Zhang, S., Kim, M.-H., Aieta, F., She, A., Mansuripur, T., Gabay, I., et al. (2016) High Efficiency Near diffraction-Limited Mid-Infrared Flat Lenses Based on Metasurface Reflect arrays. Optics Express, 24, 18024-18034. https://doi.org/10.1364/OE.24.018024

[18] Wei, T.C., et al. (2018) Broadband Achromatic Metasurface-Refractive Optics. Nano Letters, 18, 12.

[19] Overvig, A., Yu, N. and Shrestha, S. (2017) Broadband Achromatic Metasurface Lenses. Conference on Lasers and Electro-Optics, San Jose, 14-19 May 2017, Paper FM1H.3. https://doi.org/10.1364/CLEO QELS.2017.FM1H.3

[20] Shrestha, S., Overvig, A.C., Lu, M., Stein, A. and Yu, N. (2018) Broadband Achromatic Dielectric Metalenses. Light. Science \& Applications, 7, Article No. 85. https://doi.org/10.1038/s41377-018-0078-x

[21] Chen, W.T., Zhu, A.Y. and Capasso, F. (2020) Flat Optics with Dispersion-Engineered Metasurfaces. Nature Reviews Materials, 5, 604-620. https://doi.org/10.1038/s41578-020-0203-3

[22] Pfeiffer, C. and Grbic, A. (2013) Metamaterial Huygens' Surfaces: Tailoring Wave Fronts with Reflectionless Sheets. Physical Review Letters, 110, Article ID: 197401. https://doi.org/10.1103/PhysRevLett.110.197401

[23] Achouri, K. and Caloz, C. (2017) Electromagnetic Metasurfaces: Synthesis, Realizations and Discussions. In: Shamonina, E., Ed., World Scientific Handbook of Metamaterials and Plasmonics: Electromagnetic Metamaterials, Vol. 1, World Scientific, Singapore, 199-247. https://doi.org/10.1142/9789813228696 0005 
[24] Achouri, K., Salem, M.A. and Caloz, C. (2015) Improvement of Metasurface Continuity Conditions. 2015 International Symposium on Antennas and Propagation (ISAP), Hobart, 9-12 November 2015, 1-3.

[25] Mohammadi Estakhri, N. and Alù, A. (2016) Wave-Front Transformation with Gradient Metasurfaces. Physical Review X, 6, Article ID: 041008. https://doi.org/10.1103/PhysRevX.6.041008

[26] Arbabi, A. and Faraon, A. (2017) Fundamental Limits of Ultrathin Metasurfaces. Scientific Reports, 7, Article No. 43722. https://doi.org/10.1038/srep43722 